Premaksillada nadir

bilinen bir anatomik

oluşum: Canalis sinuosus

\section{A rare known anatomical structure in the remaxilla: Canalis sinuosus}

\author{
Uzm. Dt. Melike Güleç \\ Necmettin Erbakan Üniversitesi, Diş Hekimliği Fakül- \\ tesi, Ağız, Diş ve Çene Radyolojisi A.D., Konya \\ Orcid ID: 0000-0002-8616-2101
}

\section{Prof. Dr. Sevgi Özcan}

Necmettin Erbakan Üniversitesi, Diş Hekimliği Fakültesi, Ağız, Diş ve Çene Radyolojisi A.D., Konya

Orcid ID: 0000-0002-2349-9292

\section{Prof. Dr. Kaan Orhan}

Ankara Üniversitesi, Diş Hekimliği Fakültesi, Dentomaksillofasiyal Radyoloji A.D., Ankara

Orcid ID: 0000-0001-6768-0176

\section{Doç. Dr. Melek Taşsöker}

Necmettin Erbakan Üniversitesi, Diş Hekimliği Fakültesi, Ağız, Diş ve Çene Radyolojisi A.D., Konya

Orcid ID: 0000-0003-2062-5713

Geliş tarihi: 28 Ağustos 2020

Kabul tarihi: 12 Ocak 2021

doi: 10.5505/yeditepe.2021.82997

\section{Yazışma adresi:}

Melek Taşsöker

Necmettin Erbakan Üniversitesi, Diş Hekimliği Fa-

kültesi, Ağız, Diş ve Çene Radyoloji AD, Karaciğan

mahallesi Ankara cd 74/A Karatay KONYA

Tel: +90332-220-0025

E-posta: dishekmelek@gmail.com

\section{ÖZET}

Amaç: Çalışmanın amacı canalis sinuosus (CS) olarak bilinen maksillar anterior bölgedeki anatomik yapının konik ışınlı bilgisayarlı tomografi (KIBT) ile incelenmesidir.

Gereç ve Yöntem: 258 bireye ait (116 erkek, 142 kadın) KIBT görüntüleri retrospektif olarak taranmış, CS varlığı incelenmiştir. Alveolar kemik seviyesinde sonlanan en az bir kanalın görülmesi durumunda CS var olarak kodlanmıştır. CS ile yaş, cinsiyet ve bulunduğu konum arasındaki ilişki belirlenmiştir. Verilerin analizi için SPSS V.21 yazılımı (IBM Corp., Armonk, NY, USA) kullanılmış olup $p<0.05$ seviyesinde anlamlı kabul edilmiştir.

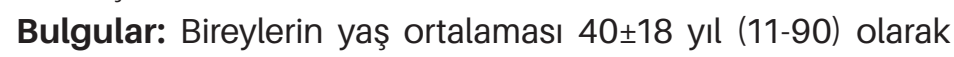
hesaplanmıştır. Çalışmada KIBT kaydı incelenen 258 bireyin $\% 70,2$ 'sinde (182 birey) en az bir tarafta alveolar kemik seviyesinde CS görülmüştür. 258 hastada toplam izlenen CS sayıSI 339 'dur. CS varlığı ile yaş ve cinsiyet arasında bir ilişki tespit edilememiştir ( $p>0.05$ ). CS'nin konumu en yüksek oranda (\%60.9) bilateraldir $(p=0.000, p<0.01)$.

Sonuç: Anterior maksillanın cerrahi öncesi KIBT incelemeleri, CS gibi (\%70.2) anatomik varyasyonların sebep olabileceği komplikasyonların önlenmesinde büyük önem arz etmektedir.

Anahtar kelimeler: Canalis sinuosus, maksilla, KIBT. SUMMARY

Aim: The aim of the study was to examine the anatomical variation in the anterior region, known as canalis sinuosus (CS), by cone-beam computed tomography (CBCT).

Materials and Methods: The СBCT images of 258 individuals (116 males, 142 females) were retrospectively scanned and the presence of CS was examined. CS is coded as present if at least one channel terminating at the alveolar bone level is seen. The relationship between CS and age, gender and location was determined. SPSS V.21 software (IBM Corp., Armonk, NY, USA) was used for data analysis and $p<0.05$ was considered significant.

Results: The mean age of the individuals was calculated as $40 \pm 18$ years (11-90). In 70.2\% (182 individuals) of 258 individuals whose KIBT records were examined in the study, CS was observed at the level of alveolar bone on at least one side. The total number of monitored CS in 258 patients was 339. There was no relationship between the presence of CS and age and gender ( $p>0.05)$. The position of CS is most bilateral $(60.9 \%)(p=0.000, p<0.01)$.

Conclusion: Preoperative CBCT examinations of the anterior maxilla are of great importance in the prevention of complications caused by anatomic variations such as CS (70.2\%).

Keywords: Canalis sinuosus, maxilla, СBCT. 


\section{GíRiş}

Maksillofasial cerrahide intraoperatif ve postoperatif komplikasyonların önlenmesinde bölge anatomisinin varyasyonları ile birlikte bilinmesi önemlidir. Konvansiyonel radyograflarda bile kolaylıkla teşhis edilebilen nazopalatin kanal, insisiv foramen ve nasal fossa, premaksilla da olarak adlandırılan anterior maksillada yer alan önemli anatomik yapılardandır. ${ }^{1}$ Canalis sinuosus (CS) bu anatomik yapılar kadar bilinmese de, cerrahi açıdan en az bu yapılar kadar önemlidir ve çoğu anatomi kaynağında bile detaylarıyla açıklanmamıştır. ${ }^{2}$ CS, ilk olarak 1930 'lu yıllarda literatüre girmiştir. ${ }^{3}$

Trigeminal sinirden ayrıan maksiller sinirin bir dalı olan infraorbital sinir, infraorbital kanal boyunca ilerlerken posterior, orta ve anterior superior alveolar (ASA) dallara ayrilır. İnfraorbital foramenin yaklaşık $25 \mathrm{~mm}$ gerisindeki infraorbital kanalın lateralinden çıkan yaklaşık olarak $2 \mathrm{~mm}$ çapındaki kemik kanalı, kurvatürlü yapısından dolayı CS olarak isimlendirilmiştir. ${ }^{3,4}$ Içinde arter ve venlerle birlikte kanin, kesici ve komşu yumuşak dokuları innerve eden ASA sinir bulunur. ${ }^{5}$ Rutin konvansiyonel radyograflarda tanımlanması güç olan CS, infraorbital kanalın lateralinden orbita tabanına iner, antrumun anterior duvarında mediale doğru kıvrılarak anterior nazal açıklığa kadar ilerler ve nazal açıklık ve burun tabanı çevresinde aşağı doğru tünel oluşturur. Bu noktada, CS genellikle maksiller kesici ve köpek dişlerinin palatinalindeki alveolar kemikte sonlanan, aksesuar kanal (AK) adı verilen anatomik varyasyonlar gösterir. ${ }^{3} \mathrm{Bu}$ varyasyon hakkında bilgi sahibi olmayan diş hekimleri CS'nin AK'larını endodontik orijinli periapikal radyolusensilerle karıştırabilmektedir. ${ }^{6}$

Konik ışınlı bilgisayarlı tomografi (KIBT) düşük maliyeti ile radyasyon dozu, yüksek çözünürlüğü ve kesitsel görüntü sunabilmesi ile geniş bir kitle tarafından kabul görmüştür. ${ }^{7-9}$ Konvansiyonel yöntemlerin aksine maksillofasial bölgenin multiplanar düzlemde detaylı bir biçimde incelenmesine olanak tanır. ${ }^{10-12}$ Literatürde, CS ile ilgili yayınların birçoğunun KIBT üzerinde rastlantısal olarak karşılaşılan vaka raporları olduğu tespit edilmiş olup, CS görülme sıkığı ve varyasyonları ile ilgili sınırlı sayıda araştırma olduğu gözlenmiştir. Bu çalışmanın amacı çapı 0.5 mm'den büyük olan, alveolar kemik seviyesinde sonlanan CS'nin görülme sıklığı ve konumunun yaşa ve cinsiyete göre dağılımının konik ışınlı bilgisayarlı tomografi (KIBT) yardımı ile incelenmesidir.

\section{GEREÇ VE YÖNTEM}

\section{Örneklem seçimi ve Çalışma dizaynı}

Bu çalışma Necmettin Erbakan Üniversitesi Diş Hekimliği Fakültesi, Ağız, Diş ve Çene Radyolojisi Ana Bilim Dalı arşivinde yer alan, 2013-2019 yılları arasında çeşitli tanısal gerekçelerle çekilmiş KIBT kayıtlarının retrospektif olarak taranmasıyla gerçekleştirilmiştir. Araştırma için gerekli etik onay Necmettin Erbakan Üniversitesi Diş Hekimliği Fakül- tesi İlaç ve Tıbbi Cihaz Dışı Araştırmalar Etik Kurulu'ndan (\#2020/01) alınmıştır. Çalışmada 11-90 yaş aralığında toplam 258 bireyin (116 erkek, 142 kadın) KIBT görüntü kaydı incelenmiştir. Maksiller anterior bölgede patoloji varlığı, dudak-damak yarığı gibi kraniyofasiyal-gelişimsel anomaliler, kraniyofasiyal travmaların tespit edildiği (vida-plak bulunan) görüntüler çalışma dışında bırakılmış olup; yeterli tanısal kaliteye sahip, artefakt bulunmayan görüntü kayıtları Ağız, Diş ve Çene Radyolojisi uzmanı olan iki gözlemci tarafından ayrı ayrı değerlendirilmiştir. CS tespitinde gözlemciler arası tam tutarlı uyum elde edilmiştir $(\mathrm{K}=1)$.

\section{Görüntülerin değerlendirilmesi}

Çalışmamızda infraorbital sinirin inferior ya da lateralinden çıkan, nazal pyriform açıklığı antero-infero-medial yönde geçerek aksesuar dalı alveolar kemik seviyesinde sonlanan en az bir aksesuar kanalın görülmesi durumunda CS var olarak kodlanmıştır. CS'ler aksiyal, sagittal ve koronal KIBT kesitlerinde incelenerek, ${ }^{13,14}$ unilateral-bilateral olarak sınıflandırılmıştır (Resim 1-3). Kanal varlığını düşündüren ancak $0.5 \mathrm{~mm}$ 'nin altında çap ölçümüne sahip oluşumlar değerlendirme dışında bırakıımıştı. ${ }^{14}$

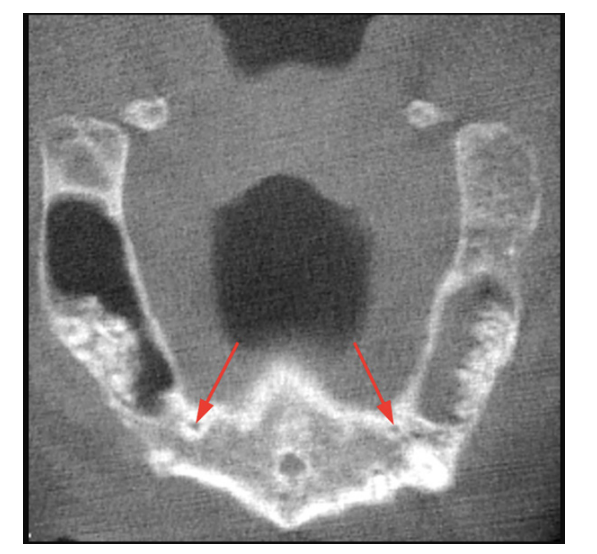

Resim1. Aksiyal KIBT kesitinde bilateral gözlenen CS.

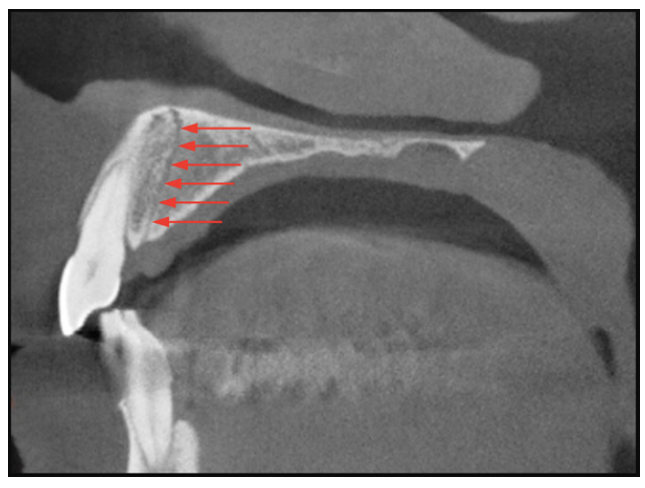

Resim2. Sagittal kesitte gözlenen CS

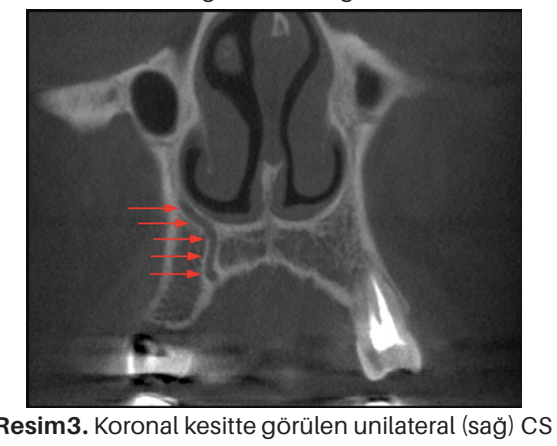




\section{Görüntüleme prosedürü}

Görüntülerin elde edilmesi için Morita 3D Accuitomo 170 (J Morita MFG Corp. Kyoto, Japan) cihazı kullanılmıştır. Çalışmada kullanılan 100x100 mm görüntüleme alanına sahip görüntüler [field of view (FOV)], $250 \mu \mathrm{m}$ voksel boyutu, 17,5 saniye ışınlama süresinde, $5 \mathrm{~mA}$ ve $90 \mathrm{kVp}$ ışınlama parametreleri kullanılarak alınmıştır. Tüm taramalar ve parametreler üreticinin tavsiye ettiği protokole göre belirlenmiştir. Görüntüler, 3.25 GB RAM ve Windows XPTM Professional operating system işlemcili $2.66 \mathrm{GHz}$ Intel Xeon bilgisayar ve 2,560×1,600 piksel çözünürlüğe sahip 27" Dell U2711HTM monitör (U2711HTM; Dell, Round Rock, TX, USA) kullanılarak incelenmiş ve değerlendirmeler için i-Dixel (J Morita MFG Corp. Kyoto, Japan) yazılımı kullanılmıştır.

\section{İstatistiksel analiz}

Verilerin analizi için SPSS V.21 yazılımı (IBM Corp., Armonk, NY, USA) kullanılmıştır. Çalışmadaki tüm parametreler için tanımlayıcı istatistik hesaplamaları (ortalama, standart sapma) yapılmıştır. CS ile cinsiyet ve bulunduğu konum arası ilişkilerin saptanmasında ki-kare testi, yaş ile CS arasındaki ilişkinin belirlenmesinde Mann-Whitney U testi kullanılmış olup, $\mathrm{p}<0.05$ seviyesinde anlamlı kabul edilmiştir.

\section{BULGULAR}

Çalışmaya dahil edilen 258 bireyin yaş ortalaması $40 \pm 18$ yıl (11-90) olarak hesaplanmıştır. Kadınların (142 birey) yaş ortalaması 37.75 (12-90), erkeklerin (116 birey) yaş ortalaması 43.68 (11-87) yıldır.

Çalışmada KIBT kaydı incelenen 258 bireyin \%70.2'sinde (182 birey) en az bir tarafta alveolar kemik seviyesinde CS görülmüştür. 258 hastada toplam izlenen CS sayıSı 339'dur. (Tablo 1).

CS'nin konumu en yüksek oranda (\%60.9) bilateraldir $(p=0.000, p<0.01$ ) (Tablo 1). Cinsiyet ile CS'nin bulunduğu konum arasında istatistiksel olarak anlamlı ilişki saptanmamıştır ( $p>0.05)$.

Tablo 1. CS'nin bulunduğu konuma göre dağılımı

Tablo 1. CS'nin bulunduğu konuma göre dağılımı

\begin{tabular}{lcccccc}
\hline CS & Kadın & Erkek & $\begin{array}{c}\text { Toplam } \\
\text { Birey }\end{array}$ & $\begin{array}{c}\text { Toplam } \\
\text { CS }\end{array}$ & \\
\hline Yok & 41 & 35 & 76 & 29,5 & 0 & \\
Sağ & 5 & 4 & 9 & 3,5 & 9 & $p=0.000$ \\
Sol & 7 & 9 & 16 & 6,2 & 16 & \\
Bilateral & 89 & 68 & 157 & 60,9 & 314 & \\
Toplam & 142 & 116 & 258 & 100,0 & 339 \\
\hline
\end{tabular}

CS varlığı ile yaş ve cinsiyet arasında bir ilişki tespit edilememiştir ( $p>0.05$ ) (Tablo 2). CS saptanan tüm bireylerin yaş ortalaması 40.16 (11-87) yıldır. CS görülen kadınlarda 35.68 (15-83), erkeklerde 45.95 (11-87) yıldır.
Tablo 2. CS'nin cinsiyete göre dağılımı

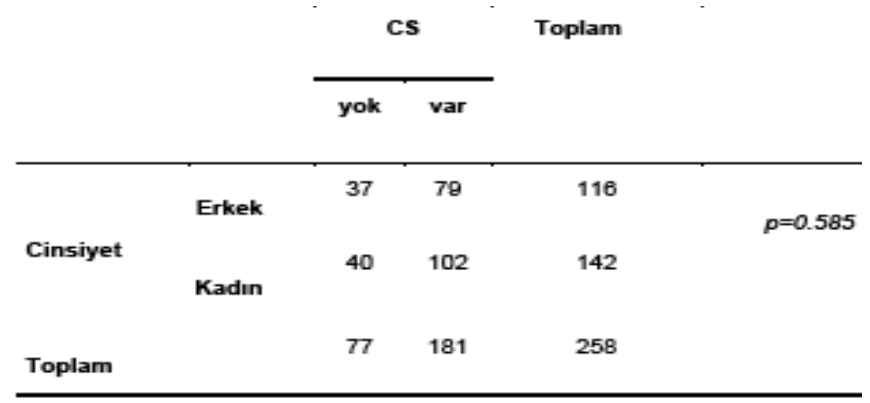

\section{TARTIŞMA}

Anterior maksillada gerçekleştirilen ortognatik cerrahi, Le Fort I ameliyatları, implant cerrahisi gibi işlemlerde, ${ }^{15}$ kist-tümör operasyonlarında, ${ }^{2}$ supernumere ve gömülü diş çekimlerinde, endodontik ve periradiküler cerrahi işlemler sırasında, ${ }^{16} \mathrm{CS}$ ile ilişkili nörovasküler demete hasar verilmesi sonucunda kanin bölgesinde geçici ya da daimi parestezi, kan kaybı, osteointegrasyonda başarısızık, ${ }^{15}$ post-operatif ağr ${ }^{14}$ gibi komplikasyonlar görülebilmektedir. Diş hekimlerinin dental işlemler sırasında karşılaşabilecekleri bu tür riskleri öngörebilmek adına çalışmamızın amacı maksiller anterior bölgede yer alan CS'nin sıklığının KIBT ile araştırılmasıdır.

$\mathrm{KIBT}, \mathrm{CS}$ incelemelerinde kullanılabilecek en iyi inceleme yöntemidir. ${ }^{15,17}$ Neves ve ark. ${ }^{18}$ bildirdikleri vakada, panoramik radyografide $C S^{\prime}$ nin, üst lateral diş kökünden nazal kaviteye seyreden dar bir radyolüsent hat olarak görülebildiğini ancak KIBT ile bu hattın CS olduğunu doğrulayabildiklerini belirtmişlerdir. Etöz ve ark. ${ }^{13}$ çalışmalarında KIBT ile tespit ettikleri 31 CS'den 5 'inin panoramik radyografilerinde radyolusent görünümlü kanalı KIBT ile doğruladıklarını ancak CS'nin ortalama çapı 2 mm'den dar olduğu için bunun iki boyutlu görüntüleme metodları ile oldukça zor olduğu bildirilmişlerdir. Shelley ve ark.6 ise bildirdikleri vakada kanin bölgesinden alınan periapikal

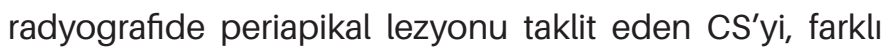
açıdan aldıkları ikinci bir radyografi ile doğrulayabildiklerini belirtmişlerdir. Maksillada sonlanan CS, anterior kesici, kanin, birinci premolar ve nazopalatin kanal çevresi bölgelere foramenlerle açılabilmekte ve ortalama çapının $1.4 \mathrm{~mm}$ olduğu belirtilmektedir (Resim 4). ${ }^{19} \mathrm{CS}$ normal bir anatomik yapı olmakla birlikte anterior palatinal bölgeye açııması yeterince tanımlanmamış preoperatif cerrahi öncesi dikkatle incelenmesi gereken anatomik bir varyasyon olarak değerlendirilmektedir. ${ }^{19}$ 


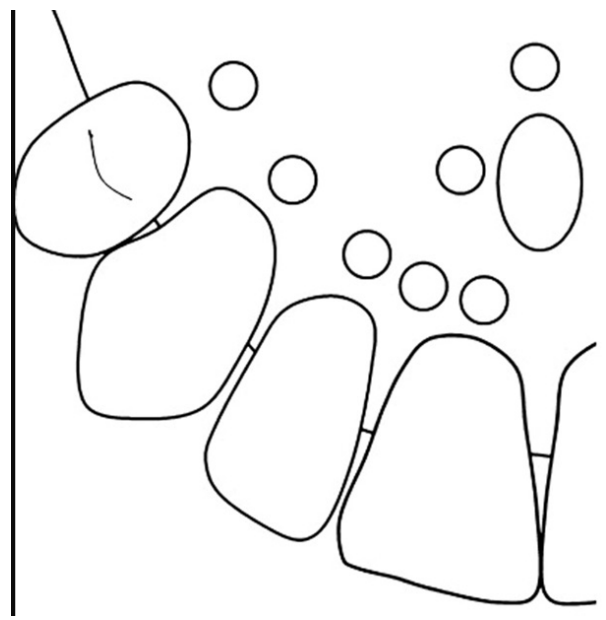

Resim 4. Maksilla anterior bölgede CS'ye ait foramenlerin olabilecek yerleşim bölgeleri (Oliveira-Santos ve ark.19)

Çalışmamızda KIBT kaydı incelenen 258 bireyin \%70.2'sinde (182 birey) en az bir tarafta alveolar kemik seviyesinde CS görülmüştür. Pubmed üzerinden yaptığımız taramada, literatürde bilgimiz dahilinde CS konusunda yürütülen, 12 adet çalışmanın bulgularına göre (Tablo 3) KIBT ile yürütülen çalışmalarda elde edilen görülme sıklıları \%4.3713\%1005 arasında çok geniş bir aralıkta seyretmektedir. Bu geniş aralığın en uç değerlerini Türk toplumunda yürütülen iki adet çalışma oluşturmaktadır.5,13 Ülkemizde CS konusunda yürütülen çalışmalarda elde edilen CS değerleri sırasılyla \%4.37,, $\% 34.7,{ }^{20} \% 70.8,{ }^{2}$ ve $\% 100^{5 \prime}$ dür. Tablo $3^{\prime}$ te belirtilen İsveç, ${ }^{21}$ Brezilya, ${ }^{22}$ Rusya ${ }^{23}$ gibi diğer toplumlardaki CS değerleri de birbirinden farklılık göstermektedir. Aynı toplum içindeki ve farklı popülasyonlardan elde edilen değerlerdeki radikal farklılıklar, CS'nin radyolojik tanımımının her çalışmada farklı planlanmış olması ve kullanılan voksel büyüklüklerinin varyasyonu ile ilişkilendirilmektedir. ${ }^{13}$ Voksel boyutunun küçük olması bu ince kanalın görüntülenmesinde büyük öneme sahiptir. ${ }^{13,18}$ CS'nin terminal sonlanma yeri nazal kavitede, maksiller sinüste ya da alveolar krette diş kökleri çevresinde olabilmektedir. Çalışmamızda yalnızca maksillar alveolar kret seviyesinde sonlanma gösteren CS'nin sıklığı araştırılmıştır. Bunun yanı sıra çalışmamızda çapı $0.5 \mathrm{~mm}$ ve üzeri ölçümlenebilen CS'ler incelenmiş olup,14,201 mm ve üzerindeki kanalları değerlendirmeye alan araştırmaların ${ }^{13,19,24}$ sonuçları da farklılığın sebebi olarak düşünülebilir. Ek olarak bu tür anatomik varyasyonların frekanslarında etnik-coğrafik yapının da etkili olduğu öne sürülmektedir. ${ }^{19}$
Tablo 3. Literatürdeki CS araştırmaları

\begin{tabular}{|c|c|c|c|c|}
\hline Araştırmaci & Yil & Incelenen KIBT sayısI & CS, $n,(\%)$ & Popülasyon \\
\hline Von Arx ve ark.24 & 2013 & 176 & $97(55.1)$ & Tsviçre \\
\hline Oliveirr-Santos ve ark. ${ }^{10}$ & 2013 & 178 & $28(15.7)$ & Brezilya \\
\hline Wanzeler ve ark. ${ }^{25}$ & 2015 & 100 & $88(88)$ & Brezilya \\
\hline Machado ve ark. ${ }^{21}$ & 2016 & 1000 & $521(52.1)$ & Isvec \\
\hline Manhaes ve ark. ${ }^{22}$ & 2016 & 500 & $181(36.2)$ & Brezilya \\
\hline Ghandourah ve ark. ${ }^{14}$ & 2017 & 219 & $144(65.75)$ & Almanya \\
\hline Gurler ve ark. ${ }^{5}$ & 2017 & 111 & $111(100)$ & Tarkiye \\
\hline Orhan ve ark. ${ }^{2}$ & 2018 & 1460 & $1034(70.8)$ & Tarkiye \\
\hline Anatoly ve ark. ${ }^{23}$ & 2019 & 150 & $101(67)$ & Rusya \\
\hline Etoz ve Yilmaz ${ }^{19}$ & 2019 & 480 & $21(4.37)$ & Tarkiye \\
\hline Aoki ve ark." & 2020 & 200 & $133(66.5)$ & Brezilya \\
\hline Tomnukçu ve Köse $e^{2 J}$ & 2020 & 326 & $113(34.7)$ & Torkiye \\
\hline Bu çalışmma & 2020 & 258 & $182(70.2)$ & Tarkiye \\
\hline
\end{tabular}

Bulgularımız CS'nin yaş ve cinsiyet ile ilişkisi olmadığını göstermiştir. De Oliveira-Santos ve ark. ${ }^{19}$, von Arx ve ark., ${ }^{24}$ Wanzeler ve ark. ${ }^{25}$ tarafından yürütülen çalışmaların sonuçları da bu çalışmamızla uyumludur. Bu konuya ilişkin, 2019 yılında Ferlin ve ark. ${ }^{15}$ tarafından yürütülen sistematik literatür derlemesinde de CS'nin yaş ve cinsiyetten bağımsız bir anatomik yapı olduğu bildirilmiştir. Çalışmamızda CS'nin \%60.9 oranında bilateral görüldüğü ve bunun istatistiksel olarak anlamlı olduğu tespit edilmiştir. Gurler ve ark. ${ }^{5} 111$ hasta inceledikleri çalışmalarında tüm KIBT görüntülerinde bilateral CS gözlemlediklerini bildirmişlerdir. Wanzeler ve ark. ${ }^{25} \% 88$, Aoki ve ark. ${ }^{17}$ ise $\% 54.4$ oranında olmak üzere CS'yi çoğunlukla bilateral gözlemlemişlerdir. CS tanımlamada farklı kanal çaplarının temel alınmasının unilateral-bilateral dağılım konusunda farklı sonuçlar oluşturduğu öne sürülmektedir. ${ }^{5}$

\section{SONUÇ}

Maksillar anterior bölgeye açılan CS, yaş ve cinsiyetten bağımsız bir yapı olup \%70.2 oranında gözlenmiştir. Bilateral görülme sıklığı yüksek bulunduğundan maksillar anterior bölgede gerçekleştirilecek cerrahi öncesinde KIBT ile bu yapının her iki maksillar bölgede araştırılması doğabilecek komplikasyonları önleyebilir. 


\section{KAYNAKLAR}

1. Tanaka, R. Hayashi T, Ohshima $H$, Ida-Yonemochi $H$, Kenmotsu $\mathrm{S}$ et al. CT anatomy of the anterior superior alveolar nerve canal: a macroscopic and microscopic study. Oral Radiol 2011;27:93-97.

2. Orhan, K, Gorurgoz C, Akyol M, Ozarslanturk S, Avsever $\mathrm{H}$. An anatomical variant: evaluation of accessory canals of the canalis sinuosus using cone beam computed tomography. Folia Morphol (Warsz) 2018;77:551-557.

3. Jones FW. The anterior superior alveolar nerve and vessels. J Anat 1939;73:583-591.

4. de Oliveira Santos C, Rubira Bullen IR, Monteiro SA, León JE, Jacobs R. Neurovascular anatomical variations in the anterior palate observed on CBCT images. Clin Oral Implants Res 2013;24:1044-1048.

5.Gurler G, Delilbasi C, Ogut EE, Aydin K, Sakul U. Evaluation of the morphology of the canalis sinuosus using cone-beam computed tomography in patients with maxillary impacted canines. Imaging Sci Dent 2017;47:69-74.

6. Shelley A, Rushton V, Horner K. Radiography: Canalis sinuosus mimicking a periapical inflammatory lesion. British Dent J 1999;186:378.

7. Allareddy V, Vincent SD, Hellstein JW, Qian F, Smoker WR et al. Incidental findings on cone beam computed tomography images. Int J Dent 2012(2012).

8. Price JB, Thaw KL, Tyndall DA, Ludlow JB, Padilla RJ. Incidental findings from cone beam computed tomography of the maxillofacial region: a descriptive retrospective study. Clin Oral Implants Res 2012;23:1261-1268.

9. Warhekar S, Nagarajappa S, Dasar PL, Warhekar AM, Parihar A et al. Incidental findings on cone beam computed tomography and reasons for referral by dental practitioners in indore city (mp). J Clin Diagn Res 2015;9:ZC2124.

10. Greenstein G, Tarnow D. The mental foramen and nerve: clinical and anatomical factors related to dental implant placement: a literature review. J Periodontol 2006;77:1933-1943.

11. Imada TSN, Fernandes LM, Centurion BS, de Oliveira-Santos $\mathrm{C}$, Honorio $\mathrm{HM}$ et al. Accessory mental foramina: prevalence, position and diameter assessed by cone beam computed tomography and digital panoramic radiographs. Clin Oral -Implants Res 2014;25:e94-e99.

12. Mraiwa $N$, Jacobs $R$, Moerman $P$, Lambrichts I, van Steenberghe $D$ et al. Presence and course of the incisive canal in the human mandibular interforaminal region: two-dimensional imaging versus anatomical observations. Surg Radiol Anat 2003;25:416-423.

13. Etöz M, Yilmaz S. Anterior Palatal Açıklığı Olan Canalis Sinuosus Varyasyonları. Turkiye Klinikleri J Dent Sci 2019;25:241-247.

14. Ghandourah AO, Rashad A, Heiland M, Hamzi BM, Friedrich RE. Cone-beam tomographic analysis of cana- lis sinuosus accessory intraosseous canals in the maxilla. Ger Med Sci 2017;15:Doc 20.

15. Ferlin R, Pagin BSC, Yaedú RYF. Canalis sinuosus: a systematic review of the literature. Oral Surg Oral Med Oral Pathol Oral Radiol 2019;127:545-551.

16. Arruda JA, Silva P, Silva $R$, Alvares $P$, Silva $L$ et al. Dental Implant in the Canalis Sinuosus: A Case Report and Review of the Literature. Case Rep Dent 2017;2017:4810123. 17. Aoki R, Massuda M, Zenni LTV, Fernandes KS. Canalis sinuosus: anatomical variation or structure? Surg Radiol Anat 2020;42:69-74.

18. Neves FS, Crusoe-Souza M, Franco LC, Caria PH, Bonfim-Almeida $P$ et al. Canalis sinuosus: a rare anatomical variation. Surg Radiol Anat 2012;34:563-566.

19. de Oliveira-Santos $C$, Rubira-Bullen IR, Monteiro SA, León JE, Jacobs R. Neurovascular anatomical variations in the anterior palate observed on CBCT images. Clin Oral Implants Res 2013;24:1044-1048.

20. Tomrukçu DN, Köse TE. Assesment of accessory branches of canalis sinuosus on CBCT images. Med Oral Patol Oral Cir Bucal 2020;25:e124-e130.

21. Machado VC, Chrcanovic BR, Felippe MB, Manhães Júnior LRC, de Carvalho PSP. Assessment of accessory canals of the canalis sinuosus: a study of 1000 cone beam computed tomography examinations. Int $\mathrm{J}$ Oral Maxillofac Surg 2016;45:1586-1591.

22. Manhães Júnior LR, Villaça-Carvalho MF, Moraes ME, Lopes SL, Silva MB et al. Location and classification of Canalis sinuosus for cone beam computed tomography: avoiding misdiagnosis. Braz Oral Res 2016;30:e49.

23. Anatoly A, Sedov Y, Gvozdikova E, Mordanov O, Kruchinina $L$ et al. Radiological and Morphometric Features of Canalis Sinuosus in Russian Population: Cone-Beam Computed Tomography Study. Int J Dent 2019:2453469.

24. von Arx T, Lozanoff $S$, Sendi P, Bornstein MM. Assessment of bone channels other than the nasopalatine canal in the anterior maxilla using limited cone beam computed tomography. Surg Radiol Anat 2013;35:783-790.

25. Wanzeler AM, Marinho CG, Alves Junior SM, Manzi FR, Tuji FM. Anatomical study of the canalis sinuosus in 100 cone beam computed tomography examinations. Oral Maxillofac Surg 2015;19:49-53. 